\title{
Systemic inflammation in childhood obesity: circulating inflammatory mediators and activated $\mathrm{CD}^{++}$monocytes
}

\author{
H. S. Schipper • R. Nuboer • S. Prop • H. J. van den Ham • \\ F. K. de Boer • Ç. Kesmir • I. M. H. Mombers • \\ K. A. van Bekkum • J. Woudstra • J. H. Kieft • \\ I. E. Hoefer • W. de Jager • B. Prakken • \\ M. van Summeren • E. Kalkhoven
}

Received: 28 March 2012 / Accepted: 15 June 2012 / Published online: 18 July 2012

(C) Springer-Verlag 2012

\begin{abstract}
Aims/hypothesis In adults, circulating inflammatory mediators and activated $\mathrm{CD} 14^{++}$monocytes link obesity to its metabolic and cardiovascular complications. However, it is largely unknown whether these inflammatory changes already occur in childhood obesity. To survey inflammatory changes during the early stages of obesity, we performed a comprehensive analysis of circulating inflammatory mediators, monocyte populations and their function in childhood obesity.

Methods In lean and obese children aged 6 to 16 years $(n=$ 96), 35 circulating inflammatory mediators including adipokines were measured. Hierarchical cluster analysis of the inflammatory mediator profiles was performed to investigate associations between inflammatory mediator clusters and clinical variables. Whole-blood monocyte phenotyping
\end{abstract}

Electronic supplementary material The online version of this article (doi:10.1007/s00125-012-2641-y) contains peer-reviewed but unedited supplementary material, which is available to authorised users.

H. S. Schipper $\cdot$ S. Prop · J. Woudstra $\cdot$ E. Kalkhoven $(\bowtie)$ Department of Metabolic Diseases,

University Medical Center Utrecht,

Room STR3.217, Universiteitsweg 100,

3584 CG Utrecht, the Netherlands

e-mail: E.Kalkhoven@umcutrecht.nl

H. S. Schipper - I. M. H. Mombers • K. A. van Bekkum •

J. H. Kieft $\cdot$ W. de Jager $\cdot$ B. Prakken

Department of Pediatric Immunology and Center for Molecular and Cellular Intervention, Wilhelmina Children's Hospital, University Medical Center Utrecht,

Utrecht, the Netherlands

\section{R. Nuboer}

Department of Pediatrics, Meander Medical Center,

Amersfoort, the Netherlands and functional testing with the toll-like receptor 4 ligand, lipopolysaccharide, were also executed.

Results First, next to leptin, the circulating mediators chemerin, tissue inhibitor of metalloproteinase 1, EGF and TNF receptor 2 were identified as novel inflammatory mediators that are increased in childhood obesity. Second, cluster analysis of the circulating mediators distinguished two obesity clusters, two leanness clusters and one mixed cluster. All clusters showed distinct inflammatory mediator profiles, together with differences in insulin sensitivity and other clinical variables. Third, childhood obesity was associated with increased $\mathrm{CD} 14^{++}$monocyte numbers and an activated phenotype of the $\mathrm{CD} 14^{++}$monocyte subsets.

Conclusions/interpretation Inflammatory mediator clusters were associated with insulin resistance in obese and lean

H. J. van den Ham

Department of Virology, Erasmus Medical Center,

Rotterdam, the Netherlands

F. K. de Boer · Ç. Kesmir

Department of Theoretical Biology and Bioinformatics, Utrecht

University,

Utrecht, the Netherlands

I. E. Hoefer

Laboratory for Experimental Cardiology, University Medical

Center Utrecht,

Utrecht, the Netherlands

M. van Summeren

Department of Pediatrics, Wilhelmina Children's Hospital,

University Medical Center Utrecht,

Utrecht, the Netherlands 
children. The activation of $\mathrm{CD} 14^{++}$monocyte subsets, which is associated with increased development of atherosclerosis in obese adults, was also readily detected in obese children. Our results indicate that inflammatory mechanisms linking obesity to its metabolic and cardiovascular complications are already activated in childhood obesity.

Keywords Adipokines · Childhood obesity · Inflammation · Insulin resistance $\cdot$ Monocytes

$\begin{array}{ll}\text { Abbreviations } & \\ \text { ALT } & \text { Alanine-aminotransferase } \\ \text { BMI-SD } & \text { BMI SD for age and sex } \\ \text { BsP } & \text { Bootstrapping probability } \\ \text { CCL } & \text { Chemokine (C-C motif) ligand } \\ \text { CCR } & \text { Chemokine (C-C motif) receptor } \\ \text { CX3CR1 } & \text { Chemokine (C-X3-C motif) receptor } 1 \\ \text { CXCL } & \text { Chemokine (C-X-C motif) ligand } \\ \text { CXCR2/5 } & \text { Chemokine (C-X-C motif) receptor 2/5 } \\ \text { EN-RAGE } & \text { Extracellular newly identified RAGE bind- } \\ & \text { ing protein } \\ \text { FABP-4 } & \text { Fatty acid binding protein } 4 \\ \text { GM-CSF } & \text { Granulocyte-macrophage colony- } \\ & \text { stimulating factor } \\ \text { HGF } & \text { Hepatic growth factor } \\ \text { hsCRP } & \text { High-sensitivity C-reactive protein } \\ \text { IP-10 } & \text { IFN-gamma-induced protein } 10 \\ \text { LPS } & \text { Lipopolysaccharide } \\ \text { M-CSF } & \text { Macrophage colony-stimulating factor } \\ \text { MCP-1 } & \text { Monocyte chemotactic protein-1 } \\ \text { MIA } & \text { Multiplex immunoassay } \\ \text { MIF } & \text { Macrophage migration inhibitory factor } \\ \text { MIP-1 } \alpha & \text { Macrophage inflammatory protein } 1 \alpha \\ \text { PAI-1 } & \text { Plasminogen activator inhibitor 1 } \\ \text { RBP-4 } & \text { Retinol binding protein 4 } \\ \text { SAA-1 } & \text { Serum amyloid A } \\ \text { sCD14 } & \text { Soluble CD14 } \\ \text { sICAM } & \text { Soluble intercellular adhesion molecule } \\ \text { sVCAM } & \text { Soluble vascular cell adhesion molecule } \\ \text { TIMP-1 } & \text { Tissue inhibitor of metalloproteinase 1 } \\ \text { TLR-4 } & \text { Toll-like receptor } 4 \\ \text { TNF-R2 } & \text { TNF receptor 2 } \\ \text { VEGF } & \text { Vascular endothelial growth factor } \\ & \end{array}$

\section{Introduction}

Obesity induces local inflammation in adipose tissue [1, 2]. As adipokines, i.e. inflammatory mediators produced by adipose tissue, are secreted into the circulation, this local obesity-induced inflammation in adipose tissue is conveyed to other sites in the body, ranging from cardiovascular tissue to circulating immune cells [3]. The resulting low-grade systemic inflammation is a pivotal link between obesity, type 2 diabetes and cardiovascular disease [4, 5]. In obese adults, low-grade inflammation is characterised by higher levels of inflammatory adipokines such as leptin, which is an important risk marker for the development of type 2 diabetes [6]. Furthermore, monocytes in obese adults are in a pro-inflammatory state $[7,8]$. Monocytes can be divided into three populations, based on their levels of $\mathrm{CD} 14$ and $\mathrm{CD} 16$ : classical $\left(\mathrm{CD} 14^{++} \mathrm{CD} 16\right)$, intermediate $\left(\mathrm{CD} 14^{++} \mathrm{CD} 16^{+}\right)$and non-classical $\left(\mathrm{CD} 14^{+} \mathrm{CD} 16^{++}\right)$[9-11]. Interestingly, greater numbers and activation of the $\mathrm{CD} 14^{++}$ populations were associated with hyperglycaemia and increased atherosclerosis in obese adults [12-14]. Taken together, circulating inflammatory mediators, such as adipokines, and activated $\mathrm{CD} 14^{++}$monocytes seem to act as inflammatory agents linking obesity to its metabolic and cardiovascular complications.

Whereas most of the knowledge on obesity-induced inflammation comes from studies in adults, childhood obesity provides an excellent opportunity to study inflammatory changes during the early stages of obesity, without confounding lifestyle habits such as smoking and co-existing inflammatory conditions like arthritis [15]. Earlier studies on small sets of circulating mediators showed greater levels of inflammatory mediators, including high-sensitivity C-reactive protein (hsCRP), leptin, IL-6 and IL-8, together with decreased levels of the insulin-sensitising adipokine, adiponectin, and higher leucocyte numbers in obese children than in lean controls [15-18]. These studies indicated that low-grade systemic inflammation is already present during childhood obesity. Considering the link between low-grade inflammation and both diabetes and cardiovascular disease, inflammatory changes at a young age may provide potential avenues for early detection and prevention of these complications $[19,20]$.

Here, we used two novel approaches to perform an indepth analysis of inflammatory mediator profiles in childhood obesity and to study the involvement of monocyte subpopulations in low-grade systemic inflammation. First, we compared plasma inflammatory mediator profiles of obese children with those of lean controls. For this, 35 inflammatory mediators, including adipokines, were measured using a recently developed multiplex immunoassay (MIA) [21]. We also conducted cluster analysis of the inflammatory mediators to see whether clinically different obese and lean control groups can be distinguished on the basis of their mediator profiles. Second, circulating monocytes in obese children and lean controls were phenotyped in detail, using a recently published flow cytometry procedure [22], and submitted to functional testing with the toll-like receptor 4 (TLR-4) ligand, lipopolysaccharide (LPS). Taken together, this is the first comprehensive study of systemic inflammation in childhood obesity to address circulating inflammatory mediator profiles and monocyte activation status. 


\section{Methods}

Participants This cross-sectional study included 96 children aged 6 to 16 years, all patients of the Paediatric Outpatient Department of the Meander Medical Center (Amersfoort, the Netherlands). The BMI and BMI SD for age and sex (BMI-SD) were calculated for all children, based on results of the Fifth Dutch Growth Study (2008-2010) [23]. Childhood obesity was defined as BMI-SD $>2.5$, which roughly corresponds to the international definition of obesity as BMI $>30 \mathrm{~kg} / \mathrm{m}^{2}$ projected to 18 years of age $[23,24]$. The population of 96 children consisted of 30 healthy lean children (BMI-SD -2.0 to 2.0), 60 age- and sex-matched obese children (BMI-SD >2.5) (Table 1), and six other children who either had a BMI-SD between 2 and 2.5, or could not be ageand sex-matched (electronic supplementary material [ESM] Table 1). Most of the analyses were performed with data from all 96 children. To compare lean and obese children, only the age and sex-matched groups (30 lean children vs 60 obese children) were used.

Exclusion criteria for all participants were: acute or chronic inflammatory and/or infectious conditions (e.g. asthma, Morbus Pfeiffer), endocrine disorders (e.g. hypothyroidism), growth disorders (e.g. growth hormone deficiency) and intoxication (smoking, drug use). Written informed consent was obtained from all children and/or their parents. The study was approved by the Institutional Medical Ethical Review Board (METC 09/217 K).

Clinical variables For bioelectrical impedance measurements (total body fat \%), a foot-hand bio-impedance analyser was used in accordance with the manufacturer's instructions (Model BIA 101; Akern, Florence, Italy) [25]. Blood pressure was measured in participants while seated and after a 5 min rest, using an automated oscillometric method (Dinamap; GE Healthcare, Amersham, UK) with an appropriately sized cuff. The lowest reading of three measurements was used. Blood pressure was normalised for age, height and sex, and expressed as a percentile. Waist circumference was measured to the nearest $1 \mathrm{~mm}$ in light expiration at the midpoint between the lowest rib and the iliac crest, in accordance with previous studies [26].

Blood samples Routine laboratory testing included: fasting glucose, fasting insulin, $\mathrm{HbA}_{1 \mathrm{c}}$, hsCRP, lipids and liver enzymes. For the other analyses, blood samples were taken in sodium-heparin tubes between 08:00 and 10:00 hours

Table 1 Clinical characteristics

\begin{tabular}{|c|c|c|c|c|}
\hline \multirow[b]{2}{*}{ Variable } & \multicolumn{2}{|l|}{ Lean controls } & \multicolumn{2}{|l|}{ Obese children } \\
\hline & Boys & Girls & Boys & Girls \\
\hline$n$ & 14 & 16 & 27 & 33 \\
\hline Age (years) & $11.3 \pm 2.8$ & $11.5 \pm 3.5$ & $12.1 \pm 2.6$ & $12.4 \pm 2.7$ \\
\hline $\mathrm{BMI}^{\mathrm{S}} \mathrm{SD}^{\mathrm{a}}$ & $0.0 \pm 1.1$ & $0.6 \pm 0.9$ & $3.7 \pm 0.6^{* * *}$ & $3.3 \pm 0.4^{\dagger \dagger \dagger}$ \\
\hline Waist circumference $(\mathrm{cm})$ & $62 \pm 8$ & $64 \pm 9$ & $90 \pm 12^{* * *}$ & $86 \pm 11^{\dagger \dagger \dagger}$ \\
\hline Total body fat $(\%)$ & $25.3 \pm 7.1$ & $30.0 \pm 4.5$ & $39.2 \pm 5.1^{* * *}$ & $40.7 \pm 3.0^{\dagger \dagger \dagger}$ \\
\hline QUICKI & $0.44(0.41-0.44)$ & $0.37(0.34-0.45)$ & $0.33(0.30-0.34)^{* * *}$ & $0.32(0.31-0.33)^{\dagger \dagger \dagger}$ \\
\hline Systolic blood pressure $(\%)^{b}$ & $48.5 \pm 29.3$ & $61.3 \pm 31.4$ & $79.7 \pm 19.7^{* * *}$ & $77.6 \pm 24.6$ \\
\hline Diastolic blood pressure $(\%)^{\mathrm{b}}$ & $38.8 \pm 21.4$ & $46.4 \pm 27.0$ & $48.4 \pm 28.7$ & $52.6 \pm 27.7$ \\
\hline Total cholesterol (mmol/l) & $3.9 \pm 0.9$ & $4.3 \pm 0.7$ & $4.2 \pm 0.9$ & $4.2 \pm 0.7$ \\
\hline HDL-cholesterol (mmol/l) & $1.4 \pm 0.3$ & $1.7 \pm 0.9$ & $1.2 \pm 0.3^{*}$ & $1.2 \pm 0.2 \dagger$ \\
\hline LDL-cholesterol (mmol/l) & $2.2 \pm 0.7$ & $2.4 \pm 0.6$ & $2.5 \pm 0.8$ & $2.5 \pm 0.7$ \\
\hline Triacylglycerol (mmol/l) & $0.6(0.4-0.7)$ & $0.8(0.5-1.1)$ & $1.0(0.6-1.4)^{*}$ & $0.9(0.7-1.3)$ \\
\hline AST (U/l) & $28(25-37)$ & $25(20-31)$ & $28(23-32)$ & $23(19-28)$ \\
\hline ALT (U/l) & $19(17-22)$ & $14(12-17)$ & $24(19-38)^{*}$ & $18(14-30)$ \\
\hline hsCRP (mg/l) & $0.15(0.15-0.48)$ & $0.33(0.16-0.73)$ & $1.70(0.78-3.20)^{* * *}$ & $2.05(0.88-3.8)^{\dagger \dagger \dagger}$ \\
\hline
\end{tabular}

Normally distributed data are shown as mean $\pm \mathrm{SD}$, non-parametric data as median (interquartile range); for clinical characteristics of the six children who either had a BMI-SD between 2 and 2.5, or could not be age- and sex-matched, see ESM Table 1

${ }^{\mathrm{a}} \mathrm{SD}$ scores, normalised for age and sex

${ }^{\mathrm{b}}$ Percentiles, normalised for age, height and sex

${ }^{*} p<0.05$ (male lean vs male obese), $\dagger p<0.05$ (female lean vs female obese), ${ }^{* * *} p<0.001$ (male lean vs male obese) and ${ }^{\dagger \dagger} p<0.001$ (female lean vs female obese)

AST, aspartate aminotransferase 
following an overnight fast. For inflammatory mediator profiling $(n=96)$, heparinised blood samples were centrifuged at $150 \mathrm{~g}$ for $10 \mathrm{~min}$, after which plasma was aliquotted and stored at $-80^{\circ} \mathrm{C}$ until analysis. For monocyte phenotyping ( $n=83,30$ lean, 49 age- and sex-matched obese children, four extra children) and functional studies ( $n=66,25$ lean, 41 age- and sex-matched obese children), whole-blood staining for flow cytometry and whole-blood stimulation with the TLR-4 ligand, LPS, were performed immediately.

Inflammatory mediator profiling Plasma sample preparation was performed exactly as described previously [21, 27]. Inflammatory mediator levels were measured with an MIA as described recently [21]. The MIA shows a high sensitivity compared with regular ELISAs, although we cannot rule out the possibility that ELISAs may incidentally show a better sensitivity [21]. Plasma samples were measured undiluted for most of the mediators. For the measurement of mediators naturally occurring in very high concentrations, 100 or 1,000 times dilutions were used. Adipsin, cathepsin $\mathrm{S}$, chemerin, leptin, plasminogen activator inhibitor-1 (PAI1), retinol binding protein 4 (RBP-4), resistin, serum amyloid A (SAA-1), tissue inhibitor of metalloproteinase-1 (TIMP-1), thrombopoietin, soluble intercellular adhesion molecule (sICAM) and soluble vascular cell adhesion molecule (sVCAM) were measured in a 100 times dilution, and adiponectin and soluble CD14 (sCD14) in a 1,000 times dilution.

Monocyte phenotyping The monocyte phenotyping protocol was adapted from Heimbeck et al [22] and performed in the dark at $4^{\circ} \mathrm{C}$. In short, $100 \mu \mathrm{l}$ whole blood was incubated for 20 min with titrated antibody mixes of: CD14 pacific blue (Biolegend, San Diego, CA, USA), HLA-DR PE-Cy7 (Biolegend), CD16 APC (Caltag, Buckingham, UK) and CD3 Percp-Cy5.5 (Biolegend), together with either CD62L FITC (Biolegend) and CD11b PE (BD Biosciences, Heidelberg, Germany), or chemokine (C-C motif) receptor 5 (CCR5) FITC (BD Biosciences) and CCR2 PE (RnD, Minneapolis, $\mathrm{MN}$, USA), or Chemokine (C-X3-C motif) receptor 1 (CX3CR1) FITC and CXCR2 PE (both from Biolegend); alternatively, isotype control antibodies were added. After the staining, samples were analysed with a flow cytometer (FACS Canto II; BD Biosciences). Monocytes were gated on the basis of their forward or sideward scatter properties and CD14/CD16 staining, after exclusion of CD3-positive and HLA-DR-negative cells. Monocyte surface marker levels were measured and quantified as the median fluorescence intensity for all surface markers.

Monocyte functional studies For monocyte functional studies, we tested the TLR-4 response of obese children and lean control monocytes, using a protocol adapted from Scholtes et al [28]. In short, $0.5 \mathrm{ml}$ whole blood was stimulated for $4 \mathrm{~h}$ with $100 \mathrm{ng} / \mathrm{ml}$ of the Escherichia coli-derived TLR-4 ligand, LPS (Sigma-Aldrich, St Louis, MO, USA). Subsequently, blood samples were centrifuged at $150 \mathrm{~g}$ for $10 \mathrm{~min}$, after which plasma was aliquotted and stored at $-80^{\circ} \mathrm{C}$ until analysis. IL- 6 and TNF- $\alpha$ levels in the plasma were measured with the MIA described above.

Statistical analyses Differences between obese children and controls were studied in the matched obese and control groups, and calculated with an independent-sample Student's $t$ test for normally distributed data or with a MannWhitney $U$ test for non-parametric comparisons. Correlations between two variables were assessed as a Pearson correlation coefficient for normally distributed data and as a Spearman correlation coefficient for non-parametric comparisons.

For regression analysis of the inflammatory mediators with BMI-SD, the age- and sex-dependence of the inflammatory mediators was determined by comparing the levels of inflammatory mediators between boys and girls, and performing correlation analysis of the inflammatory mediators with age. Next, simple correlation analysis with BMISD (Pearson, Spearman) was performed for inflammatory variables not depending on age or sex. For inflammatory variables depending on age and/or sex, partial correlations with BMI-SD were calculated, while controlling for age and sex.

For inflammatory mediator profiling (Table 2) and monocyte phenotyping (ESM Table 2), the $p$ values were corrected for multiple testing using Benjamini and Hochberg's false discovery rate correction for multiple testing [29].

Non-supervised hierarchical cluster analysis was performed for the whole study population and for all inflammatory mediators, using a method adapted from van den Ham et al [30]. As quality control testing revealed that participants and mediators with $>10 \%$ missing values clustered together and interfered with the analysis, these participants $(n=8$, seven obese children, one other child) and mediators (IFN- $\gamma$, IL-6, TNF- $\alpha$, RBP-4, SAA-1, granulocyte-macrophage colonystimulating factor [GM-CSF], macrophage inflammatory protein $1 \alpha$ [MIP-1a], extracellular newly identified RAGE binding protein [EN-RAGE], chemokine [C-X-C motif] ligand 8 [CXCL8]) were excluded from the cluster analysis. Where values were missing in $<10 \%$ of participants, measurement values below the detection limit were set to the lowest detection limit. The data were log-transformed and normalised per mediator to have a mean of 0 (i.e. $y i=x i-\frac{1}{n} \sum_{i=0}^{n} x i$ ) and a range of -1 to 1 (i.e. $\left.z i=\frac{y i}{\max _{1 \leq i \leq n}\left|y_{i}\right|}\right)$. Subsequently, the normalised data were hierarchically clustered using Ward's (minimum variance) linkage and 1- $\rho$ as distance measure, 
Table 2 Circulating inflammatory mediators

\begin{tabular}{|c|c|c|c|c|c|}
\hline \multirow[b]{2}{*}{ Variable } & \multirow[b]{2}{*}{ Units } & \multicolumn{2}{|l|}{ Obesity status } & \multicolumn{2}{|c|}{ Regression (BMI-SD) } \\
\hline & & Lean & Obese & Age/sex correction & Correlation coefficient \\
\hline \multicolumn{6}{|l|}{ Cytokines } \\
\hline IFN- $\gamma^{\mathrm{d}}$ & $\mathrm{pg} / \mathrm{ml}$ & $0.95(0.95-1.96)$ & $0.95(0.95-0.95)$ & None & -0.112 \\
\hline $\mathrm{IL}^{-6^{\mathrm{d}}}$ & $\mathrm{pg} / \mathrm{ml}$ & $0.13(0.13-62.1)$ & $0.13(0.13-52.2)$ & None & -0.032 \\
\hline IL-10 & $\mathrm{pg} / \mathrm{ml}$ & $11.9(9.6-19.7)$ & $11.4(9.3-15.8)$ & Age & 0.012 \\
\hline IL-18 & $\mathrm{pg} / \mathrm{ml}$ & $313(235-375)$ & $373(313-476)^{*}$ & None & 0.217 \\
\hline $\mathrm{TNF}-\alpha^{\mathrm{c}}$ & $\mathrm{pg} / \mathrm{ml}$ & $2.3(1.1-4.2)$ & $1.3(0.01-2.6)$ & None & $-0.305^{*}$ \\
\hline \multicolumn{6}{|l|}{ Adipokines } \\
\hline Adiponectin $^{\mathrm{b}}$ & $\mu \mathrm{g} / \mathrm{ml}$ & $33.5 \pm 10.8$ & $29.5 \pm 10.0$ & Age & -0.134 \\
\hline Adipsin ${ }^{\mathrm{a}}$ & $\mathrm{ng} / \mathrm{ml}$ & $615(595-643)$ & $605(575-624)$ & None & -0.179 \\
\hline Cathepsin S & $\mathrm{ng} / \mathrm{ml}$ & $56.4 \pm 11.7$ & $60.0 \pm 11.2$ & None & $0.240^{*}$ \\
\hline Chemerin & $\mu \mathrm{g} / \mathrm{ml}$ & $2.8 \pm 0.4$ & $3.0 \pm 0.5^{*}$ & None & $0.273^{*}$ \\
\hline FABP- $4^{\mathrm{a}}$ & $\mathrm{ng} / \mathrm{ml}$ & $23.6(20.5-27.9)$ & $24.0(21.5-27.0)$ & None & -0.060 \\
\hline HGF & $\mathrm{pg} / \mathrm{ml}$ & $282(223-339)$ & $365(260-523)^{*}$ & None & 0.154 \\
\hline Leptin & $\mathrm{ng} / \mathrm{ml}$ & $123(111-159)$ & $298(210-452)^{* * *}$ & Sex & $0.627^{* * *}$ \\
\hline Omentin & $\mathrm{pg} / \mathrm{ml}$ & $3.8(3.3-4.4)$ & $4.0(3.5-4.5)$ & None & 0.032 \\
\hline PAI- $1^{\mathrm{a}}$ & $\mu \mathrm{g} / \mathrm{ml}$ & $168 \pm 45.3$ & $151 \pm 41.0$ & None & -0.181 \\
\hline RBP- $4^{\mathrm{c}}$ & $\mu \mathrm{g} / \mathrm{ml}$ & $153(139-180)$ & $156(143-168)$ & Age & -0.048 \\
\hline Resistin $^{a}$ & $\mathrm{ng} / \mathrm{ml}$ & 919 (901-963) & 925 (890-985) & None & 0.037 \\
\hline SAA- $1^{\mathrm{d}}$ & $\mu \mathrm{g} / \mathrm{ml}$ & $68.4(35.4-100)$ & $40.0(24.7-100)$ & None & -0.212 \\
\hline TIMP-1 & $\mathrm{ng} / \mathrm{ml}$ & $23.0(7.6-26.2)$ & $25.6(21.7-30.3)^{*}$ & None & $0.238^{*}$ \\
\hline Thrombopoietin & $\mu \mathrm{g} / \mathrm{ml}$ & $12.3(12.0-12.9)$ & $12.3(11.7-12.8)$ & None & -0.178 \\
\hline \multicolumn{6}{|l|}{ Monocytes } \\
\hline $\mathrm{GM}^{-\mathrm{CSF}^{\mathrm{d}}}$ & $\mathrm{pg} / \mathrm{ml}$ & $0.12(0.12-2.7)$ & $0.12(0.12-0.12)$ & None & -0.143 \\
\hline $\mathrm{M}-\mathrm{CSF}^{\mathrm{a}}$ & $\mathrm{pg} / \mathrm{ml}$ & $56.0(44.5-72.2)$ & $50.1(35.9-69.2)$ & None & -0.212 \\
\hline $\mathrm{MIF}^{\mathrm{b}}$ & $\mathrm{pg} / \mathrm{ml}$ & $877(311-1,262)$ & $1,083(591-1,696)$ & None & 0.119 \\
\hline MCP-1/CCL2 ${ }^{\mathrm{a}}$ & $\mathrm{pg} / \mathrm{ml}$ & $408 \pm 144$ & $380 \pm 185$ & None & -0.150 \\
\hline MIP-1a/CCL3 $3^{\mathrm{c}}$ & $\mathrm{pg} / \mathrm{ml}$ & $49.4(20.0-76.3)$ & $40.5(12.9-84.2)$ & None & -0.025 \\
\hline MIP-1b & $\mathrm{pg} / \mathrm{ml}$ & $77.9(63.3-95.8)$ & $95.5(67.2-144)$ & None & 0.128 \\
\hline $\mathrm{sCD} 14^{\mathrm{b}}$ & $\mu \mathrm{g} / \mathrm{ml}$ & $7.4(6.0-9.6)$ & $7.7(6.5-9.9)$ & None & 0.209 \\
\hline \multicolumn{6}{|l|}{ Chemotaxis/other } \\
\hline $\mathrm{EGF}^{\mathrm{a}}$ & $\mathrm{pg} / \mathrm{ml}$ & $57.2 \pm 38.6$ & $90.5 \pm 61.1^{*}$ & None & $0.228 *$ \\
\hline EN-RAGE ${ }^{\mathrm{c}}$ & $\mathrm{pg} / \mathrm{ml}$ & $35.5(2.28-64.8)$ & $6.6(0.12-48.5)$ & None & -0.160 \\
\hline CXCL8/IL-8 ${ }^{\mathrm{c}}$ & $\mathrm{pg} / \mathrm{ml}$ & $44.5(23.5-69.7)$ & $64.9(34.4-211)^{*}$ & Age & 0.122 \\
\hline IP-10 & $\mathrm{pg} / \mathrm{ml}$ & $291(199-388)$ & $395(291-471)^{*}$ & None & 0.206 \\
\hline TNF-R $1^{\mathrm{a}}$ & $\mathrm{ng} / \mathrm{ml}$ & $2.6 \pm 0.7$ & $2.7 \pm 0.8$ & None & 0.042 \\
\hline TNF-R2 & $\mathrm{ng} / \mathrm{ml}$ & $2.6 \pm 0.6$ & $3.0 \pm 0.6^{*}$ & None & $0.273^{*}$ \\
\hline sICAM & $\mu \mathrm{g} / \mathrm{ml}$ & $3.0(2.8-3.2)$ & $3.1(2.9-3.3)$ & None & 0.010 \\
\hline sVCAM & $\mu \mathrm{g} / \mathrm{ml}$ & $5.1 \pm 0.5$ & $5.2 \pm 0.5$ & Age & 0.072 \\
\hline VEGF $^{\mathrm{b}}$ & $\mathrm{pg} / \mathrm{ml}$ & $113(57.6-159)$ & $156(87.0-238)$ & None & 0.104 \\
\hline
\end{tabular}

Circulating inflammatory mediator levels for age and sex-matched lean $(n=30)$ and obese $(n=60)$ children. For clarity, mediators are classified according to origin and function: classical cytokines, adipokines, mediators affecting monocyte function and mediators involved in chemotaxis/other

Normally distributed data are shown as mean $\pm \mathrm{SD}$, non-parametric data as median (interquartile range). For regression analysis with BMI-SD, inflammatory mediators of the six children who either had a BMI-SD between 2 and 2.5, or could not be age- and sex-matched were also included (total of 96 children)

Mediators for which values were missing, mostly due to undetectable levels, are indicated as follows: ${ }^{\mathrm{a}} 1-3 \%$ missing values, ${ }^{\mathrm{b}} 3-10 \%$ missing values, ${ }^{\mathrm{c}}>10 \%$ missing values, ${ }^{\mathrm{d}}>30 \%$ missing values

$* p<0.05, * * * p<0.001$

CCL, chemokine (C-C motif) ligand; FABP-4, fatty acid binding protein 4; MCP-1, Monocyte chemotactic protein-1 
where $\rho$ stands for pairwise complete Pearson correlation. To assess the robustness of mediator and patient clusters, bootstrapping $(n=10,000)$ was applied and the consensus clusters used for further analyses. Heatmaps were created by combining the mediator and study population clustering. To compare the distribution of clinical variables for obesity (BMI-SD, waist circumference, body fat percentage) and insulin sensitivity (QUICKI) between two different clusters, a Mann-Whitney $U$ test was used.

Statistical analyses were performed with SPSS 15.0 for Windows (SPSS, Chicago, IL, USA) and R, a free software environment for statistical computing and graphics [31]. Bootstrapping was performed using the Pvclust package [32].

\section{Results}

Circulating inflammatory mediators Obese children and lean controls had characteristic differences in clinical variables (Table 1). First, together with age- and sex-corrected BMI-SD, waist circumference and total body fat percentage were increased in the obese children. Second, obese boys and girls had lower insulin sensitivity than lean controls $(p<0.001)$, as determined by QUICKI, a wellestablished surrogate measure for insulin sensitivity in adults and children [33, 34]. Third, obese boys had higher systolic blood pressure corrected for age and height $(p<0.001)$, higher triacylglycerol levels $(p=0.018)$ and higher alanineaminotransferase (ALT) levels $(p=0.020)$. Finally, obese boys and girls had lower HDL-cholesterol levels $(p=0.030$ and $p=0.004$, respectively), and higher hsCRP levels $(p<0.001)$ than lean controls (Table 1).

Profiling of circulating inflammatory mediators, including adipokines, also showed characteristic differences between obese children and lean controls. As expected [35], leptin levels were more than twofold higher in obese children than in lean controls (Table 2). Furthermore, after correction for multiple testing, several novel childhood obesity-associated inflammatory mediators were identified. Plasma levels of IL-18, chemerin, hepatic growth factor (HGF), TIMP-1, EGF, CXCL8 (IL-8), IFNgamma-induced protein 10 (IP-10) and TNF receptor 2 (TNF-R2) were higher in obese children than in lean controls. For five of these mediators (leptin, chemerin, TIMP-1, EGF and TNF-R2, fold inductions 2.4, 1.1, 1.1, 1.6 and 1.2, respectively), the association with childhood obesity seemed particularly relevant, as linear regression analysis also showed a positive correlation with BMI-SD (Table 2, ESM Fig. 1).

In conclusion, next to leptin, the inflammatory mediators chemerin, TIMP-1, EGF and TNF-R2 were identified as circulating mediators that are increased in childhood obesity and correlate with BMI-SD. Differences in these mediators could not be attributed to differences in age or sex between obese children and lean controls, as the groups were ageand sex-matched and none of the five increased inflammatory mediators was correlated with age. Only leptin was correlated with sex, i.e. had higher levels in girls than in boys, as reported before [35], but this sex effect did not influence the positive correlation between leptin and BMISD (Table 2).

Cluster analysis distinguishes clinically different obese and lean control groups To identify clusters of inflammatory mediators and investigate whether obese children and controls can be distinguished via such clusters, non-hierarchical cluster analysis of the inflammatory mediator profiles was performed.

First, we identified three robust clusters of inflammatory mediators that met the requirements of bootstrapping probability (BsP) $>50 \%$ (ESM Fig. 2a). Correlation analysis on the three robust clusters was performed to verify the results (ESM Fig. 2b). As expected, all three robust clusters showed a high correlation between the inflammatory mediators: adipsin and thrombopoietin (BsP 100, $R^{2}=0.91$ ); cathepsin $\mathrm{S}$ and chemerin (BsP 57, $R^{2}=0.53$ ); and the third cluster consisting of IL-18, TNF-R2 and IP-10 (BsP 80; correlation between IL-18 and TNF-R2, $R^{2}=0.46$ and correlation between IL-18 and IP-10, $R^{2}=0.36$; ESM Fig. $2 b$ ). Taken together, cluster analysis showed three robust clusters of inflammatory mediators. Two of these clusters seemed particularly interesting (adipsin and thrombopoietin, cathepsin $\mathrm{S}$ and chemerin), as they were part of a larger cluster of inflammatory mediators, including leptin, which roughly distinguished lean and obese children, as shown in a heatmap (Fig. 1a).

Second, cluster analysis of the inflammatory mediator profiles distinguished lean and obese children, and showed five distinct participant clusters (Fig. 1a). Obese children were predominantly concentrated in clusters I and II, while clusters III and IV mainly comprised lean controls, and cluster V was mixed (Fig. 1a, g). As chemerin, leptin, TIMP-1, EGF and TNF-R2 concentrations were increased in childhood obesity and correlated with BMI-SD (Table 2), the levels of these mediators for all five clusters were studied in detail (Fig. 1b-f, ESM Fig. 3b). Furthermore, the relationships between inflammatory mediators and clinical variables were investigated (Fig. 1g-k). Comparison of the obesity clusters I and II showed that several inflammatory mediators, e.g. leptin, TIMP-1, EGF, chemerin, macrophage migration inhibitory factor (MIF) and vascular endothelial growth factor (VEGF), occurred at high levels in cluster I, but not in cluster II (Fig. 1d, e, ESM Fig. 3b). The high inflammatory mediator levels in obesity cluster I 
Fig. 1 Cluster analysis of the inflammatory mediators. (a) Hierarchical cluster analysis of the inflammatory mediator profiles was performed in 88 of the 96 children, resulting in two obesity clusters, two leanness clusters and one mixed cluster. The heatmap shows logtransformed inflammatory mediator levels for all individual children. White blocks represent missing values. (b) Distribution of adiponectin, (c) leptin, (d) TIMP-1, (e) EGF and (f) TNFR2 over the five clusters, and (g-k) over clinical characteristics as labelled. Medians are represented by a black bar. Blue and red dots represent age- and sex-matched lean $(n=30)$ and obese $(n=53)$ children, respectively. Black dots represent children who either had a BMISD between 2 and 2.5 , or could not be age- and sex-matched $(n=5) .{ }^{*} p<0.05$ a
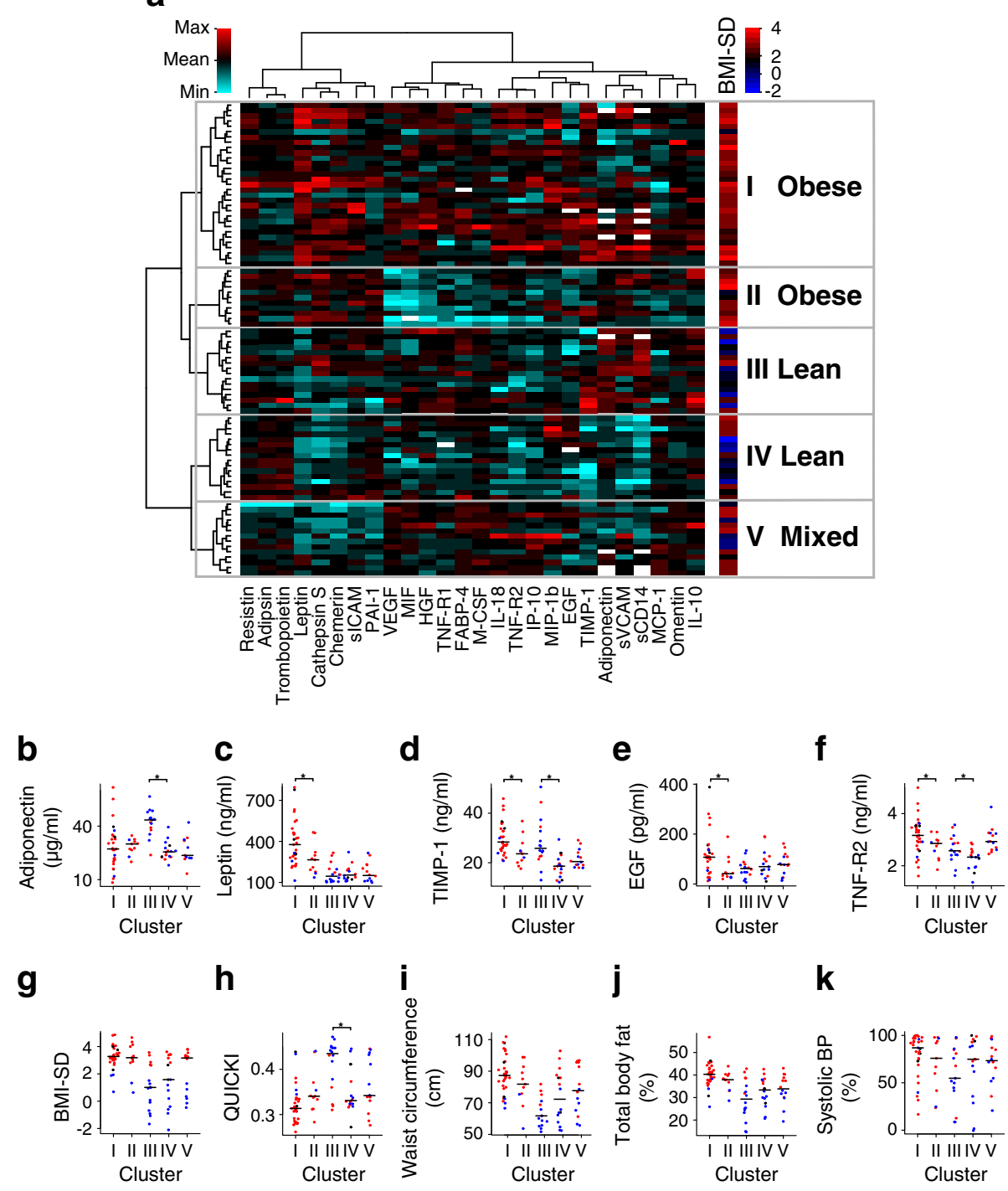

\section{k}

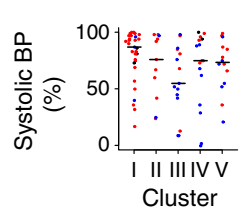

coincided with a trend towards lower insulin sensitivity (QUICKI) than that in cluster II ( $p=0.07)$ (Fig. 1h). Similarly, the inflammatory mediator profiles of leanness clusters III and IV distinguished clinically different lean control groups. Thus the lean controls in cluster III had higher levels of inflammatory mediators such as TIMP-1 and TNF-R2 (Fig. 1d and f), but mainly were conspicuous for their high levels of the insulin-sensitising adipokine, adiponectin, compared with leanness cluster IV (Fig. 1b). The high levels of adiponectin in cluster III coincided with a trend towards lower waist circumference $(p=0.09)$ and significantly higher insulin sensitivity (QUICKI, $p=0.01$ ) (Fig. 1h and i).

In summary, high levels of inflammatory mediators (leptin, TIMP-1, EGF, VEGF and MIF) coincided with a trend towards lower insulin sensitivity in obesity cluster I vs II, while high levels of insulin-sensitising adiponectin coincided with higher insulin sensitivity in leanness cluster III vs IV. In other words, obesity cluster II and leanness cluster III seem to represent healthy groups, compared with their counterparts.

Monocyte phenotyping and functional analysis To study the involvement of monocyte subsets in the low-grade inflammation occurring in childhood obesity, monocyte subtype numbers were determined and extensive monocyte phenotyping (i.e. surface marker expression) was performed on whole blood samples. First, monocytes were gated as classical $\left(\mathrm{CD} 14^{++} \mathrm{CD} 16^{-}\right)$, intermediate $\left(\mathrm{CD} 14^{++} \mathrm{CD} 16^{+}\right)$and non-classical $\left(\mathrm{CD} 14^{+} \mathrm{CD} 16^{++}\right)$monocyte subsets, in accordance with previous studies (Fig. 2a) [9, 10, 13]. Quantification of monocyte subset numbers revealed that the numbers of $\mathrm{CD} 14^{++}$classical and intermediate monocytes, but not those of non-classical monocytes correlated positively with BMI-SD (Fig. 2b). Second, the surface expression of the following adhesion factors and chemokine receptors that are involved in atherosclerosis was studied: 
CD62L, CD11b, CCR2, CCR5, CX3CR1 and chemokine (C-X-C motif) receptor 2 (CXCR2) (Fig. 2c-e) [36, 37]. Subsequently, correlations were calculated between, on the one hand, monocyte subset numbers, and adhesion factor and chemokine expression, and, on the other hand, the clinical variables BMI-SD, waist circumference, total body fat percentage, QUICKI and systolic blood pressure. Correlation coefficients for all significant correlations $(p<0.05$, without multiple testing correction) are shown in a heatmap (Fig. 2f). After correction for multiple testing, classical monocyte numbers and CD11b abundance on the classical and intermediate monocyte subsets remained as the monocyte markers most significantly correlated with BMI-SD (ESM Table 2).

To verify the increased numbers and activated phenotype of classical monocytes in obese children functionally, whole-blood samples were stimulated with the TLR-4 ligand, LPS. Although other TLR-4-proficient cells can also secrete IL- 6 and TNF- $\alpha$, monocytes account for most of the cytokine production in response to whole-blood stimulation with LPS [28], with classical monocytes secreting high levels of IL-6, while non-classical monocytes produce more TNF- $\alpha$ [10]. The IL- 6 and TNF- $\alpha$ levels after LPS stimulation therefore give an indication of the balance between classical and non-classical monocytes. Interestingly, intermediate monocytes do not have striking levels of IL-6 or TNF- $\alpha$ production upon LPS stimulation [10], but their involvement was not tested with this assay. In line with the high numbers and activated phenotype of classical monocytes observed in obese children, we observed higher IL-6 levels upon LPS stimulation in them than in lean controls, but no differences in TNF- $\alpha$ levels (Fig. $2 \mathrm{~g}$ and $\mathrm{h}$ ).

Taken together, especially classical $\left(\mathrm{CD} 14^{++} \mathrm{CD} 16^{-}\right)$ monocytes seem to be involved in the low-grade systemic inflammation seen in childhood obesity, as this subset showed increased numbers, increased CD11b levels and high IL-6 production in response to LPS in obese children compared with lean controls. While intermediate $\left(\mathrm{CD} 14^{++} \mathrm{CD} 16^{+}\right)$monocytes also exhibited greater expression of the activation marker CD11b, the non-classical $\left(\mathrm{CD} 14^{+} \mathrm{CD} 16^{++}\right)$monocytes showed no alterations in number or phenotype in obese children.

\section{Discussion}

Across diverse adult patient groups, circulating inflammatory mediators and activated $\mathrm{CD} 14^{++}$monocytes have been identified as inflammatory agents linking obesity to its metabolic and cardiovascular complications [3, 12, 13]. To the best of our knowledge, this is the first comprehensive study of inflammatory mediator profiles and monocyte populations in childhood obesity, thereby providing novel insights into the inflammatory changes that occur during the early stages of obesity.

First, next to leptin, the mediators chemerin, TIMP-1, EGF and TNF-R2 were identified as novel inflammatory mediators that are increased in childhood obesity. All of these mediators act at the crossroads of metabolism and inflammation, and have been associated with decreased insulin sensitivity in mouse models, obese adults and obese children [38-41]. Interestingly, high chemerin levels were recently associated with endothelial activation in obese children [42]. The relatively subtle differences observed by us in the levels of these circulating proteins between lean and obese individuals are in agreement with other studies [39, 42]. Local concentrations (i.e. in the target tissue) may display larger differences, but this remains to be established. Our second insight was that, in cluster analysis of the inflammatory mediators, clinically different obese and lean control groups emerged. Comparing the two obesity clusters, high levels of inflammatory mediators such as leptin, TIMP-1 and EGF coincided with a trend towards lower insulin sensitivity. In the two leanness clusters, high levels of the insulin-sensitising adipokine, adiponectin, coincided with higher insulin sensitivity. Our third insight was that childhood obesity is associated with increased classical monocyte numbers and an activated phenotype of the classical and intermediate monocyte subsets, including increased IL-6 production upon TLR-4 stimulation. Taken together, our findings reveal an association between inflammatory mediator clusters and insulin resistance, both in obese and lean control groups. Furthermore, activation of $\mathrm{CD}_{14}{ }^{++}$monocyte subsets, which is associated with increased atherosclerosis in obese adults $[12,13]$, was already readily detected in obese children.

Our comprehensive approach enabled the identification of several novel inflammatory mediators in childhood obesity. Moreover, cluster analysis showed the relevance of inflammatory mediators for important clinical variables such as insulin sensitivity (QUICKI). Nevertheless, this study has two weaknesses. First, obese children and lean controls were age- and sex-matched, but age- and sexmediated effects on inflammatory mediators could not always be excluded. The small differences in age and sex distribution between the obese and lean control clusters, though statistically non-significant, may have influenced the mediator clustering (ESM Fig. 3a). Second, the relatively small obese and lean control groups, and the wide agerange of our participants could have masked differences between lean and obese children. Thus while we report a significant correlation between chemerin and BMI-SD, an even stronger correlation was recently observed in a larger cohort with older children [42]. At the same time, subtle differences in inflammatory mediators may also 
Fig. 2 Monocyte populations in childhood obesity. (a) Gating of classical $\left(\mathrm{CD} 14^{++} \mathrm{CD} 16^{-}\right.$; green), intermediate $\left(\mathrm{CD} 14^{+}\right.$

${ }^{+} \mathrm{CD} 16{ }^{+}$; purple) and nonclassical (CD14 ${ }^{+} \mathrm{CD} 16^{++}$; red) monocyte subsets. (b) Correlation of the monocyte population numbers with BMI-SD, with significant correlations between both classical (correlation coefficient 0.270 ) and intermediate (correlation coefficient 0.220 ) monocyte numbers and BMISD. (c) Representative phenotype of the classical, (d) intermediate and (e) non-classical monocytes. Quadrants express isotype control staining. (f) Correlation coefficients between monocyte numbers (no.), monocyte phenotype (surface marker expression) and clinical variables. Only significant correlations are shown $(p<0.05)$. Monocyte phenotyping data of 30 lean and 49 age- and sexmatched obese children, and four children who either had a BMISD between 2 and 2.5 , or could not be age- and sex-matched (total 83 children) were available and used for the analyses.

RRsyst, systolic blood pressure. (g) IL-6 and (h) TNF- $\alpha$ levels upon $4 \mathrm{~h}$ of TLR-4 stimulation with LPS, performed in 25 lean and 41 obese children (total 66 children). ${ }^{*} p<0.05$
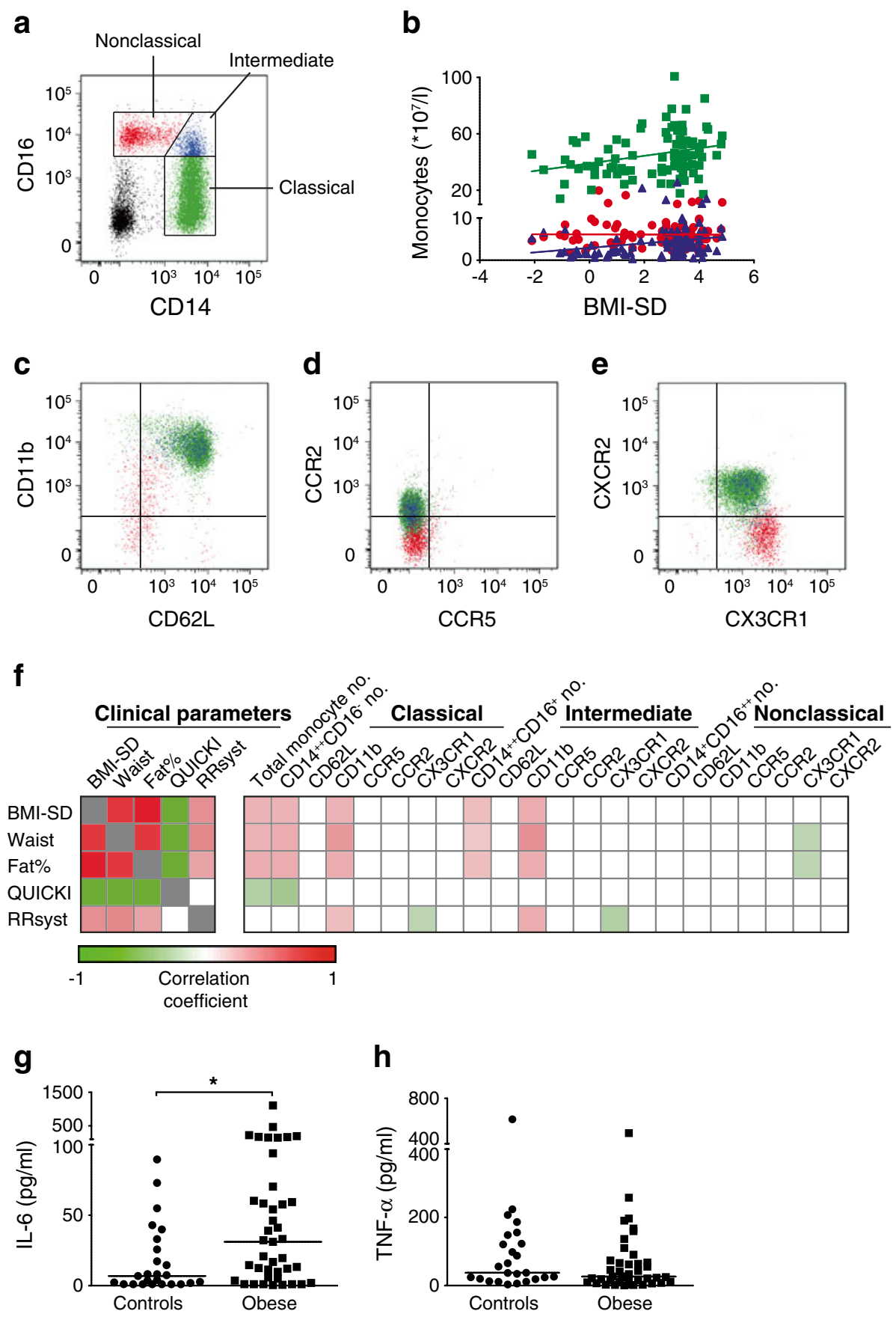

have been missed due to the relatively small groups with a wide age-range. For example, we were unable to confirm the increased IL-10 levels previously observed in obese adolescent girls [18]. Moreover, based on previous studies [43], we would also have expected lower adiponectin levels in obese children than in controls, but merely observed a trend $(p=0.09)$. Interestingly, however, cluster analysis revealed that, compared with the other clusters, leanness cluster III was striking for its high adiponectin levels, coinciding with high insulin sensitivity.
Increased numbers of circulating monocytes have been reported in obese children and adults $[8,44]$. While CD14 ${ }^{++}$ monocytes have been implicated in the development of atherosclerosis in obese adults [12-14], this is, to our knowledge, the first study showing that $\mathrm{CD} 14^{++}$monocyte numbers are already increased in childhood obesity. We also showed that $\mathrm{CD} 14^{++}$monocytes in obese children are striking for their high levels of CD11b, compared with lean controls. CD11b is an $\alpha$-integrin that plays a key role in cell-adhesive interactions and the migration of cells to inflammatory sites [45]. Recently, however, CD11b was also 
identified as a negative regulator of TLR-4-mediated inflammatory responses. In fact, $\mathrm{CD} 11 \mathrm{~b}$ abundance is upregulated upon TLR-4 stimulation [28] and inhibits inflammatory pathways downstream of TLR-4 [46]. Taken together, the high $\mathrm{CD} 11 \mathrm{~b}$ expression on $\mathrm{CD} 14^{++}$monocytes in obese children may propagate monocyte migration to inflammatory sites, such as vascular lesions or adipose tissue. Alternatively, the increased monocyte $\mathrm{CD} 11 \mathrm{~b}$ expression in childhood obesity may reflect TLR-4 stimulation and serve to inhibit TLR-4mediated inflammatory pathways. In obesity, increased translocation of bacterial LPS from the gut and spillover of saturated fatty acids can induce TLR-4 signalling [47-49] and may contribute to the observed upregulation of $\mathrm{CD} 11 \mathrm{~b}$ on $\mathrm{CD} 14^{++}$monocytes. Yet it is important to note that IL-6 secretion in response to TLR-4 stimulation is still increased in obese children (Fig. 2d). Thus, increased monocyte CD11b expression does not prevent a higher TLR-4-mediated inflammatory response in obese children. Furthermore, three intriguing questions with respect to monocyte activation in childhood obesity remain. First, while previously investigated in adults [12-14], the effects of $\mathrm{CD} 14^{++}$monocytes on vascular function in obese children requires further study. Second, definitions of the recently identified intermediate monocyte subset vary and its role in vascular disease is as yet unclear $[10,11]$. Our results suggest that intermediate monocytes are upregulated in childhood obesity and activated like classical monocytes, but the question of whether these cells fulfil a specific role in childhood obesity requires further investigation. Third, as adipokines are known to modulate leucocyte function [3], monocyte function may be affected by inflammatory mediator profiles. Indeed, we observed the highest number of intermediate monocytes in obesity cluster I, coinciding with its inflammatory mediator profile (ESM Fig. 4a). Moreover, leanness cluster III showed the lowest number of classical monocytes, coinciding with its high adiponectin levels (ESM Fig. 4a). Nevertheless, no differences in monocyte phenotype (e.g. CD11b expression) were observed between the clusters (ESM Fig. 4b), and further investigation is needed to address the interaction between adipokines and circulating monocytes in childhood obesity.

In conclusion, this study addressed two inflammatory modes of action in childhood obesity. On the one hand, inflammatory mediator clusters were found to correlate with insulin resistance in obese and lean control groups. On the other hand, activation of $\mathrm{CD} 14^{++}$monocytes, which is associated with increased atherosclerosis development in obese adults $[12,13]$, was readily detectable in childhood obesity. Thus inflammatory mediators and activated $\mathrm{CD} 14^{++}$monocytes seem to be part of the inflammatory link between obesity and its metabolic and cardiovascular complications, and may provide potential avenues for early detection and prevention of these complications.
Acknowledgements The authors thank the secretaries of the Paediatric Outpatient Department and the paediatricians of the Meander Medical Center in Amersfoort (the Netherlands) for their help with participant recruitment. We also thank the Endocrinology Laboratory (University Medical Center, Utrecht, the Netherlands) for help with laboratory measurements, and M. Hendriks and D. Vis (Department of Metabolic Diseases, University Medical Center, Utrecht) for advice on statistical methods.

Funding This research was performed within the framework of the Center for Translational Molecular Medicine (CTMM; www.ctmm.nl), project Circulating Cells (grant 01C-102), and supported by the Dutch Heart Foundation, the UMC Utrecht Vascular Prevention Project and the Wilhelmina Children's Hospital Research Fund.

Duality of interest The authors declare that there is no duality of interest associated with this manuscript.

Contribution statement HSS, RN, IEH, WJ, BP, MS and EK designed the study. HSS, RN, SP, IMHM, KAB, JW JK and WJ recruited participants, collected data and participated in the analysis of the data. HJH, FKB and CK performed statistical analyses. HSS drafted the manuscript, and all authors revised it critically for intellectual content. All authors have approved the final version.

\section{References}

1. Lumeng CN, Saltiel AR (2011) Inflammatory links between obesity and metabolic disease. J Clin Invest 121:2111-2117

2. Hotamisligil GS (2006) Inflammation and metabolic disorders. Nature 444:860-867

3. Ouchi N, Parker JL, Lugus JJ, Walsh K (2011) Adipokines in inflammation and metabolic disease. Nat Rev Immunol 11:85-97

4. Donath MY, Shoelson SE (2011) Type 2 diabetes as an inflammatory disease. Nat Rev Immunol 11:98-107

5. Rocha VZ, Libby P (2009) Obesity, inflammation, and atherosclerosis. Nat Rev Cardiol 6:399-409

6. Li S, Shin HJ, Ding EL, van Dam RM (2009) Adiponectin levels and risk of type 2 diabetes: a systematic review and meta-analysis. JAMA 302:179-188

7. Kullo IJ, Hensrud DD, Allison TG (2002) Comparison of numbers of circulating blood monocytes in men grouped by body mass index $(<25,25$ to $<30,>$ or $=30)$. Am J Cardiol 89:1441-1443

8. Ghanim H, Aljada A, Hofmeyer D, Syed T, Mohanty P, Dandona P (2004) Circulating mononuclear cells in the obese are in a proinflammatory state. Circulation 110:1564-1571

9. Ziegler-Heitbrock L (2007) The CD14+ CD16+ blood monocytes: their role in infection and inflammation. J Leukoc Biol 81:584-592

10. Wong KL, Tai JJ, Wong WC et al (2011) Gene expression profiling reveals the defining features of the classical, intermediate, and nonclassical human monocyte subsets. Blood 118:e16-e31

11. Zawada AM, Rogacev KS, Rotter B et al (2011) SuperSAGE evidence for CD14++CD16+ monocytes as a third monocyte subset. Blood 118:e50-e61

12. Poitou C, Dalmas E, Renovato $M$ et al (2011) CD14dimCD16+ and $\mathrm{CD} 14+\mathrm{CD} 16+$ monocytes in obesity and during weight loss: relationships with fat mass and subclinical atherosclerosis. Arterioscler Thromb Vasc Biol 31:2322-2330

13. Rogacev KS, Ulrich C, Blomer L et al (2010) Monocyte heterogeneity in obesity and subclinical atherosclerosis. Eur Heart $\mathrm{J}$ 31:369-376 
14. Satoh N, Shimatsu A, Himeno A et al (2010) Unbalanced M1/M2 phenotype of peripheral blood monocytes in obese diabetic patients: effect of pioglitazone. Diabetes Care 33:e7

15. Tam CS, Clement K, Baur LA, Tordjman J (2010) Obesity and low-grade inflammation: a paediatric perspective. Obes Rev 11:118-126

16. Visser M, Bouter LM, McQuillan GM, Wener MH, Harris TB (2001) Low-grade systemic inflammation in overweight children. Pediatrics 107:E13

17. Skinner AC, Steiner MJ, Henderson FW, Perrin EM (2010) Multiple markers of inflammation and weight status: crosssectional analyses throughout childhood. Pediatrics 125:e801e809

18. Tam CS, Garnett SP, Cowell CT et al (2010) IL-6, IL-8 and IL-10 levels in healthy weight and overweight children. Horm Res Paediatr 73:128-134

19. Tirosh A, Shai I, Afek A et al (2011) Adolescent BMI trajectory and risk of diabetes versus coronary disease. N Engl J Med 364:1315-1325

20. Lloyd LJ, Langley-Evans SC, McMullen S (2012) Childhood obesity and risk of the adult metabolic syndrome: a systematic review. Int J Obes (Lond) 36:1-11

21. Schipper HS, de Jager W, van Dijk ME et al (2010) A multiplex immunoassay for human adipokine profiling. Clin Chem 56:1320 1328

22. Heimbeck I, Hofer TP, Eder C et al (2010) Standardized singleplatform assay for human monocyte subpopulations: lower CD14 + CD16++ monocytes in females. Cytometry A 77:823-830

23. Schonbeck Y, Talma H, van Dommelen P et al (2011) Increase in prevalence of overweight in Dutch children and adolescents: a comparison of nationwide growth studies in 1980, 1997 and 2009. PLoS One 6:e27608

24. Cole TJ, Bellizzi MC, Flegal KM, Dietz WH (2000) Establishing a standard definition for child overweight and obesity worldwide: international survey. BMJ 320:1240-1243

25. Kondaki K, Grammatikaki E, Pavon DJ et al (2011) Comparison of several anthropometric indices with insulin resistance proxy measures among European adolescents: The Helena Study. Eur J Pediatr 170:731-739

26. Lawlor DA, Fraser A, Lindsay RS et al (2010) Association of existing diabetes, gestational diabetes and glycosuria in pregnancy with macrosomia and offspring body mass index, waist and fat mass in later childhood: findings from a prospective pregnancy cohort. Diabetologia 53:89-97

27. Verrijn Stuart AA, Schipper HS, Tasdelen I et al (2012) Altered plasma adipokine levels and in vitro adipocyte differentiation in pediatric type 1 diabetes. J Clin Endocrinol Metab 97:463-472

28. Scholtes VP, Versteeg D, de Vries JP et al (2011) Toll-like receptor 2 and 4 stimulation elicits an enhanced inflammatory response in human obese patients with atherosclerosis. Clin Sci (Lond) 121:205-214

29. Benjamini Y, Hochberg Y (1995) Controlling the false discovery rate - a practical and powerful approach to multiple testing. J Roy Stat Soc B Methodological 57:289-300

30. van den Ham HJ, de Jager W, Bijlsma JW, Prakken BJ, de Boer RJ (2009) Differential cytokine profiles in juvenile idiopathic arthritis subtypes revealed by cluster analysis. Rheumatol (Oxford) 48:899-905

31. Team RDC (2011) R: a language environment for statistical computing. R Foundation for Statistical Computing, Vienna, Austria
32. Suzuki R, Shimodaira H (2006) Pvclust: an R package for assessing the uncertainty in hierarchical clustering. Bioinformatics 22:1540-1542

33. Chen H, Sullivan G, Quon MJ (2005) Assessing the predictive accuracy of QUICKI as a surrogate index for insulin sensitivity using a calibration model. Diabetes 54:1914-1925

34. Gunczler P, Lanes R (2006) Relationship between different fastingbased insulin sensitivity indices in obese children and adolescents. J Pediatr Endocrinol Metab 19:259-265

35. Garcia-Mayor RV, Andrade MA, Rios M, Lage M, Dieguez C, Casanueva FF (1997) Serum leptin levels in normal children: relationship to age, gender, body mass index, pituitary-gonadal hormones, and pubertal stage. J Clin Endocrinol Metab 82:28492855

36. Gautier EL, Jakubzick C, Randolph GJ (2009) Regulation of the migration and survival of monocyte subsets by chemokine receptors and its relevance to atherosclerosis. Arterioscler Thromb Vasc Biol 29:1412-1418

37. Boschmann M, Engeli S, Adams F et al (2005) Adipose tissue metabolism and CD11b expression on monocytes in obese hypertensives. Hypertension 46:130-136

38. Ernst MC, Sinal CJ (2010) Chemerin: at the crossroads of inflammation and obesity. Trends Endocrinol Metab 21:660-667

39. Gupta A, Ten S, Anhalt H (2005) Serum levels of soluble tumor necrosis factor-alpha receptor 2 are linked to insulin resistance and glucose intolerance in children. J Pediatr Endocrinol Metab 18:75-82

40. Meissburger B, Stachorski L, Roder E, Rudofsky G, Wolfrum C (2011) Tissue inhibitor of matrix metalloproteinase 1 (TIMP1) controls adipogenesis in obesity in mice and in humans. Diabetologia 54:1468-1479

41. Prada PO, Ropelle ER, Mourao RH et al (2009) EGFR tyrosine kinase inhibitor (PD153035) improves glucose tolerance and insulin action in high-fat diet-fed mice. Diabetes 58:2910-2919

42. Landgraf K, Friebe D, Ullrich T et al (2012) Chemerin as a mediator between obesity and vascular inflammation in children. J Clin Endocrinol Metab 97:E556-E564

43. Stefan N, Bunt JC, Salbe AD, Funahashi T, Matsuzawa Y, Tataranni PA (2002) Plasma adiponectin concentrations in children: relationships with obesity and insulinemia. J Clin Endocrinol Metab 87:4652-4656

44. Zaldivar F, McMurray RG, Nemet D, Galassetti P, Mills PJ, Cooper DM (2006) Body fat and circulating leukocytes in children. Int J Obes (Lond) 30:906-911

45. Basoni C, Nobles M, Grimshaw A et al (2005) Inhibitory control of TGF-beta1 on the activation of Rap1, CD11b, and transendothelial migration of leukocytes. FASEB J 19:822-824

46. Han C, Jin J, Xu S, Liu H, Li N, Cao X (2010) Integrin CD11b negatively regulates TLR-triggered inflammatory responses by activating Syk and promoting degradation of MyD88 and TRIF via Cbl-b. Nat Immunol 11:734-742

47. Greiner T, Backhed F (2011) Effects of the gut microbiota on obesity and glucose homeostasis. Trends Endocrinol Metab 22:117-123

48. Shi H, Kokoeva MV, Inouye K, Tzameli I, Yin H, Flier JS (2006) TLR4 links innate immunity and fatty acid-induced insulin resistance. J Clin Invest 116:3015-3025

49. Holland WL, Bikman BT, Wang LP et al (2011) Lipid-induced insulin resistance mediated by the proinflammatory receptor TLR4 requires saturated fatty acid-induced ceramide biosynthesis in mice. J Clin Invest 121:1858-1870 